\title{
Spline Embedding for Nonlinear Dimensionality Reduction
}

\author{
Shiming Xiang ${ }^{1}$, Feiping $\mathrm{Nie}^{1}$, Changshui Zhang ${ }^{1}$, and Chunxia Zhang ${ }^{2}$ \\ ${ }^{1}$ State Key Laboratory of Intelligent Technology and Systems, \\ Department of Automation, Tsinghua University, Beijing 100084, China \\ \{xsm, nfp03, zcs\}@mail.tsinghau.edu.cn \\ 2 School of Computer Science, Software School, \\ Beijing Institute of Technology, Beijing 100081, China \\ cxzhang@bit.edu.cn
}

\begin{abstract}
This paper presents a new algorithm for nonlinear dimensionality reduction (NLDR). Smoothing splines are used to map the locally-coordinatized data points into a single global coordinate system of lower dimensionality. In this work setting, we can achieve two goals. First, a global embedding is obtained by minimizing the low-dimensional coordinate reconstruction error. Second, the NLDR algorithm can be naturally extended to deal with out-of-sample data points. Experimental results illustrate the validity of our method.
\end{abstract}

\section{Introduction}

Recently, nonlinear dimensionality reduction(NLDR) is a hot topic in machine learning. The motivation behind NLDR is to discover an informative representation hidden in high-dimensional data points. Generally, the task is to evaluate the embedding coordinates of the data points on the low-dimensional manifold.

Many algorithms have been proposed to learn a low-dimensional embedding: Isomap [1, locally linear embedding (LLE) [2], Laplacian eigenmap 3], Hessian LLE (HLLE) 4, local tangent space alignment (LTSA) 5, etc.. They are developed to embed a set of given data points. However, most of them are not capable of embedding new data points into the learned manifold in a natural way. This is known as the out-of-sample problem.

This paper presents a new algorithm for NLDR. The algorithm is developed from the conceptual framework of compatible mapping. A data point in the manifold can be represented in different local coordinate systems, but it has a single global coordinate on the low-dimensional manifold. Compatible mappings under different local coordinate systems map a data point into the low-dimensional manifold such that its global coordinate is unique. Smoothing splines are used to construct such mappings.

Our algorithm has many parallels to manifold learning by Isomap, LLE, Laplacian eigenmap, HLLE, LTSA, etc.. Most notably, the objective function is based on the reconstruction error, and a global low-dimensional embedding is achieved by solving an eigenvalue problem. 
Our algorithm can be naturally extended to deal with out-of-sample data points, due to spline interpolation. Here, a natural extension mean that the algorithm can embed new data points in way of how to treat the training data points. For most manifold learning algorithms [6], however, it is difficult to achieve this goal in such a natural way.

\section{Mappings}

The NLDR problem can be formulated as follows. Given a set of $n$ scattered data points $\boldsymbol{x}_{i} \in \mathbb{R}^{m}$ lying on a manifold $M$ embedded in a $m$-dimensional Euclidean space. The goal is to invert an underlying generative model $\boldsymbol{x}=f(\boldsymbol{y})$ to find the corresponding low-dimensional parameters (embedding coordinates) $\boldsymbol{y}_{i} \in \mathbb{R}^{d}$ such that $\boldsymbol{x}_{i}=f\left(\boldsymbol{y}_{i}\right)$, say, construct $\mathcal{Y}=\left\{\boldsymbol{y}_{i}\right\}_{i=1}^{n}$ from $\mathcal{X}=\left\{\boldsymbol{x}_{i}\right\}_{i=1}^{n}$.

\subsection{Compatible Mappings}

Generally, given a parameter space $\Omega \subset \mathbb{R}^{d}$ and a mapping $f: \Omega \mapsto \mathbb{R}^{m}$ with $m>d$, then $M=f(\Omega)$ is called a parameterized manifold embedded in $\mathbb{R}^{m}$. In view of Riemannian geometry, it can be locally coordinatized. Thus we can use local coordinates to exploit the local geometrical structures of the data. Then we face a task - the points with local coordinates should be aligned together into the low-dimensional manifold with a single global coordinate system.

Denote the neighborhood of $\boldsymbol{x}_{i}(i=1, \cdots, n)$ by $\mathcal{N}_{i}(\subset \mathcal{X} \subset M)$. According to the above analysis, for each $\mathcal{N}_{i}$, we need two mappings, $h_{i}: \mathcal{N}_{i} \rightarrow \mathcal{T}_{i}$ and $g_{i}: \mathcal{T}_{i} \rightarrow \mathcal{Y}_{i}(\subset \mathcal{Y} \subset \Omega)$. The task of $h_{i}$ is to locally coordinatize the data points in $\mathcal{N}_{i}$, while the task of $g_{i}$ is to transform the local coordinates in $\mathcal{T}_{i}$ into the global coordinates. Let $\varphi_{i}=g_{i} \circ h_{i}$ be a compound mapping of $h_{i}$ and $g_{i}$, then $\varphi_{i}$ will be used to align the data points in $\mathcal{N}_{i}$ into a global coordinate system.

To avoid alignment conflicts, we need to consider the data points in the intersections of the neighborhoods. Without loss of generality, we consider $\mathcal{N}_{1}$ and $\mathcal{N}_{2}$. For $\boldsymbol{x} \in \mathcal{N}_{1} \cap \mathcal{N}_{2}$, since it has a unique $\boldsymbol{y} \in \mathcal{Y}$, we have

$$
\varphi_{1}\left(\mathcal{N}_{1} \cap \mathcal{N}_{2}\right)=\varphi_{2}\left(\mathcal{N}_{1} \cap \mathcal{N}_{2}\right)
$$

Definition: Let $\varphi_{1}$ and $\varphi_{2}$ be two mappings. We say $\varphi_{1}$ and $\varphi_{2}$ are compatible with each other if $\varphi_{1}(\boldsymbol{x})=\varphi_{2}(\boldsymbol{x})$ holds for any $\boldsymbol{x} \in \mathcal{N}_{1} \cap \mathcal{N}_{2}$.

Now we can see that we need $n$ mappings $\left\{\varphi_{i}\right\}_{i=1}^{n}$ which are compatible with each other. It seems quite complex to construct such mappings since there are lots of constraints as eq. (1). However, we have the following proposition:

Proposition: Let $\mathcal{N}_{i}$ contains $k$ data points in $\mathcal{X}$ and denote $\mathcal{N}_{i}=\left\{\boldsymbol{x}_{i_{j}}\right\}_{j=1}^{k}$. Here, subscript $i_{j}$ stands for an index and $i_{j} \in\{1, \cdots, n\}$. For each $\varphi_{i}=g_{i} \circ h_{i}$, if $h_{i}$ is an injection and for $\boldsymbol{t}_{i}^{j}=h_{i}\left(\boldsymbol{x}_{i_{j}}\right)$, we have

$$
\boldsymbol{y}_{i_{j}}=g_{i}\left(\boldsymbol{t}_{i}^{j}\right), \quad j=1,2, \cdots, k
$$

then $\varphi_{i}, i=1, \cdots, n$, are compatible with each other. 
Since $h_{i}$ is an injection, then $\boldsymbol{t}_{i}^{1}=h_{i}\left(\boldsymbol{x}_{i_{1}}\right) \neq \boldsymbol{t}_{i}^{2}=h_{i}\left(\boldsymbol{x}_{i_{2}}\right)$ holds if $\boldsymbol{x}_{i_{1}} \neq \boldsymbol{x}_{i_{2}}$. Thus, each $\boldsymbol{x}_{i_{j}} \in \mathcal{N}_{i}$ has a unique local coordinate $\boldsymbol{t}_{i}^{j}$ in $\mathcal{T}_{i}=h_{i}\left(\mathcal{N}_{i}\right)$. Although $\boldsymbol{x}_{i_{j}}$ has different local coordinates in different coordinate systems, it has a unique global coordinate $\boldsymbol{y}_{i_{j}}$. According to eq. (2), the above proposition holds.

Based on this proposition, the constraints in eq. (1) can be naturally satisfied. Thus, we can construct $h_{i}$ and $g_{i}$ by only starting with $\mathcal{N}_{i}$.

We will treat $h_{i}$ as a linear projection to a tangent space of $M$ defined at $\boldsymbol{x}_{i}$ (subsection 2.2). In addition, smoothing splines will be used to construct $g_{i}$ so that the constraints in eq. (2) can be faithfully satisfied (subsections 2.3-2.5).

\subsection{Tangent Space Projection}

We suppose that $M$ is a smooth manifold, then the tangent space $T_{x}(M) \subset \mathbb{R}^{m}$ can be well defined at each point $\boldsymbol{x} \in M$. We use this subspace to define the local coordinates 45] and construct $h_{i}$. The steps can be summarized as follows:

First, for each data point $\boldsymbol{x}_{i} \in \mathcal{X}, i=1, \cdots, n$, we identify its $k$-nearest neighbors in Euclidean distance to construct a neighborhood $\mathcal{N}_{i}$.

Then, for each $\mathcal{N}_{i}$, we let $X_{i}=\left[\boldsymbol{x}_{i_{1}}, \boldsymbol{x}_{i_{2}}, \cdots, \boldsymbol{x}_{i_{k}}\right] \in \mathbb{R}^{m \times k}$, and perform a singular value decomposition of the centralized matrix of $X_{i}$ :

$$
X_{i}\left(I-\frac{1}{k} e \cdot e^{T}\right)=U_{i} \Sigma_{i} V_{i}^{T}, \quad i=1, \cdots, n
$$

where $I$ is a $k \times k$ identity matrix, $e=[1,1, \cdots, 1]^{T} \in \mathbb{R}^{k}, \Sigma_{i}=[\Sigma, 0]^{T} \in$ $\mathbb{R}^{m \times k}$, in which $\Sigma=\operatorname{diag}\left(\sigma_{1}, \cdots, \sigma_{k}\right), U_{i}$ contains $m$ left singular vectors, and $V_{i}$ contains $k$ right singular vectors. Finally, we get the local coordinate $\boldsymbol{t}_{i}^{j} \in \mathbb{R}^{d}$ :

$$
\boldsymbol{t}_{i}^{j}=\left(\tilde{U}_{i}\right)^{T} \cdot\left(\boldsymbol{x}_{i_{j}}-\overline{\boldsymbol{x}}_{i}\right)=\tilde{\Sigma}_{i} \cdot \boldsymbol{v}_{i}^{j}, \quad j=1, \cdots, k
$$

here $\tilde{U}_{i}$ is a $m \times d$ sub-matrix which contains the first $d$ column vectors of $U_{i}$, $\overline{\boldsymbol{x}}_{i}=\frac{1}{k} \sum_{j=1}^{k} \boldsymbol{x}_{i_{j}}, \tilde{\Sigma}_{i}=\operatorname{diag}\left(\sigma_{1}, \cdots, \sigma_{d}\right), \boldsymbol{v}_{i}^{j}$ is a $d$-dimensional vector which contains the first $d$ components of the $j$-th row of matrix $V_{i}$.

Now $h_{i}$ can be defined as a subspace projection operator from $\mathbb{R}^{m}$ to $\mathbb{R}^{d}$, i.e., $h_{i} \triangleq\left(\tilde{U}_{i}\right)^{T}$. In view of mappings, it is an injection since $\left(\tilde{U}_{i}\right)^{T}$ has full row rank.

\subsection{Interpolation Conditions}

Think momentarily of $\varphi_{i}=g_{i} \circ h_{i}$ as the inverse mapping of $f$ near point $\boldsymbol{x}_{i}$. Note that $f^{-1}$ is usually highly nonlinear and $h_{i}$ is a linear operator, which is used to exploit the locally linear geometrical structure of the data. Then it is suitable to construct $g_{i}$ as a nonlinear mapping. But $g_{i}$ should meet the $k$ constraints as formulated in eq. (2). To this end, we use nonlinear splines to construct $g_{i}$.

Now we let $Y_{i}=\left[\boldsymbol{y}_{i_{1}}, \boldsymbol{y}_{i_{2}}, \cdots, \boldsymbol{y}_{i_{k}}\right] \in \mathbb{R}^{d \times k}$ contain $k$ global coordinates of data points in $\mathcal{N}_{i}$. Denote its $r$-th row by $\left[y_{i_{1}}^{(r)}, \cdots, y_{i_{k}}^{(r)}\right], r=1, \cdots, d$. In terms of splines, we divide the constraints in eq. (2) into $d$ groups of interpolation conditions. The $r$-th group corresponds to the $r$-th coordinate components:

$$
y_{i_{j}}^{(r)}=g_{i}^{r}\left(\boldsymbol{t}_{i}^{j}\right), \quad j=1,2, \cdots, k
$$


Finally, $g_{i}$ can be constructed as a vector function, which contains $d$ splines, namely, $g_{i}=\left[g_{i}^{1}, g_{i}^{2}, \cdots, g_{i}^{d}\right]^{T}$.

\subsection{Smoothing Splines}

To be clear, we use $\boldsymbol{\theta}_{j}, z_{j}$ and $g$ to replace $\boldsymbol{t}_{i}^{j}, y_{i_{j}}^{(r)}$ and $g_{i}^{r}$ in eq. (15). Then

$$
z_{j}=g\left(\boldsymbol{\theta}_{j}\right), \quad j=1,2, \cdots, k
$$

For eq. (6), we can minimize the following objective functional:

$$
J(g)=\frac{1}{k} \sum_{j=1}^{k}\left(z_{j}-g\left(\boldsymbol{\theta}_{j}\right)\right)^{2}+J_{s}^{d}(g)
$$

where $J_{s}^{d}(g)$ is a penalty functional, which stipulates the continuity of functions. We choose to optimize the functional $J(g)$ in Sobolev space 78 . Then, a smoothing spline solution can be formulated as follows 78 :

$$
g(\boldsymbol{\theta})=\sum_{j=1}^{k} \alpha_{j} \phi_{j}(\boldsymbol{\theta})+\sum_{i=1}^{l} \beta_{i} p_{i}(\boldsymbol{\theta})
$$

where $\phi_{j}(\boldsymbol{\theta})$ is a Green's function and $p_{i}(\boldsymbol{\theta})$ is a polynomial term. All such $p_{i}(\boldsymbol{\theta})$, $i=1, \cdots, l$, constitute a basis of a polynomial space with degree less than $s$. Considering the second term in eq. (8) as a linear polynomial and denoting $\boldsymbol{\theta}=\left[\theta_{1}, \theta_{2}\right]^{T}$ in case of $d=2$, for example, then we have $p_{1}(\boldsymbol{\theta})=1, p_{2}(\boldsymbol{\theta})=\theta_{1}$ and $p_{3}(\boldsymbol{\theta})=\theta_{2}$. In this case, $l$ is equal to 3 . In addition, $\phi_{j}(\boldsymbol{\theta})=\left(\left\|\boldsymbol{\theta}-\boldsymbol{\theta}_{j}\right\|\right)^{2 s-d}$. $\log \left(\left\|\boldsymbol{\theta}-\boldsymbol{\theta}_{j}\right\|\right)$ if $d$ is even; $\phi_{j}(\boldsymbol{\theta})=\left(\left\|\boldsymbol{\theta}-\boldsymbol{\theta}_{j}\right\|\right)^{2 s-d}$ if $d$ is odd.

To avoid degeneracy, we add conditionally positive definition constraints:

$$
\sum_{j=1}^{k} \alpha_{j} \cdot p_{i}\left(\boldsymbol{\theta}_{j}\right)=0, \quad i=1, \cdots, l
$$

Now substituting the interpolation conditions into eq. (8) and eq. (9), we can get a linear system for solving the coefficients $\boldsymbol{\alpha} \in \mathbb{R}^{k}$ and $\boldsymbol{\beta} \in \mathbb{R}^{l}$ :

$$
\left(\begin{array}{cc}
K & P \\
P^{T} & 0
\end{array}\right) \cdot\left(\begin{array}{c}
\boldsymbol{\alpha} \\
\boldsymbol{\beta}
\end{array}\right)=\left(\begin{array}{c}
\boldsymbol{z} \\
0
\end{array}\right), \quad \text { i.e., } \quad A \cdot\left(\begin{array}{c}
\boldsymbol{\alpha} \\
\boldsymbol{\beta}
\end{array}\right)=\left(\begin{array}{c}
\boldsymbol{z} \\
0
\end{array}\right)
$$

where $K$ is a $k \times k$ symmetrical matrix with elements $K_{i j}=\phi\left(\left\|\boldsymbol{\theta}_{i}-\boldsymbol{\theta}_{j}\right\|\right), P$ is a $k \times l$ matrix with elements $P_{i j}=p_{i}\left(\boldsymbol{\theta}_{j}\right), \boldsymbol{z}=\left[z_{1}, \cdots, z_{k}\right]^{T}$, and $A$ is used to denote the coefficient matrix.

The spline as formulated in eq. (8) has several good properties. First, $g\left(\boldsymbol{\theta}_{j}\right)=$ $z_{j}$ holds for all $j$ when $P$ has full row rank. This can be satisfied in the work setting of NLDR. In case of $d=2$, for example, the scattered points should not be in a line. Faithfully satisfying the interpolation conditions is necessary for us to construct compatible mappings. Second, it is smooth and we can use it to interpolate new points near $\left\{\boldsymbol{\theta}_{j}\right\}_{j=1}^{k}$. This yields a mechanism to treat the out-of-samples. Third, the interpolation error can be estimated in form of $k$ interpolation values $z_{j}, j=1, \cdots, k$. Thus, we can construct an objective function. By minimizing it, a global optimal embedding can be achieved.

Actually, $J_{s}^{d}(g)$ can be approximated as $\boldsymbol{\alpha}^{T} K \boldsymbol{\alpha}=\boldsymbol{z}^{T}\left(B^{T} K B\right) \boldsymbol{z}$, , in which $B$ is the upper left $k \times k$ subblock of $A^{-1}$. By some mathematical deductions, it turns out the following interpolation error for $J(g)$ :

$$
J(g) \approx \boldsymbol{z}^{T} B \boldsymbol{z}
$$




\subsection{Mapping Local Coordinates to Global Coordinates}

The steps for constructing a vector function $g_{i}$ are summarized as follows:

(1) Use $\boldsymbol{t}_{i}^{j}$ to replace $\boldsymbol{\theta}_{j}$ in eq. (6), and calculate the coefficient matrix $A$ in eq. (10). Adding a subscript $i$, we get $A_{i}$;

(2) Use eq. (10) $d$ times for $d$ coordinate components. Actually, we have

$$
A_{i} \cdot\left(\begin{array}{c}
\boldsymbol{\alpha}_{1}, \cdots, \boldsymbol{\alpha}_{d} \\
\boldsymbol{\beta}_{1}, \cdots, \boldsymbol{\beta}_{d}
\end{array}\right)=\left(\begin{array}{c}
Y_{i}^{T} \\
0
\end{array}\right)
$$

(3) Respectively substitute coefficients $\left(\boldsymbol{\alpha}_{1}, \boldsymbol{\beta}_{1}\right), \cdots,\left(\boldsymbol{\alpha}_{d}, \boldsymbol{\beta}_{d}\right)$ into eq. (8) to construct $d$ splines: $g_{i}^{1}, \cdots, g_{i}^{d}$. Thus we obtain $g_{i}=\left[g_{i}^{1}, \cdots, g_{i}^{d}\right]^{T}$, which is used to map the local coordinates to the global coordinates.

Finally, based on eq. (11), the reconstruction error for $d$ coordinate components can be calculated as follows:

$$
e_{i}=\operatorname{tr}\left(Y_{i} B_{i} Y_{i}^{T}\right)
$$

where $t r$ is a trace operator, and $B_{i}$ is the upper left $k \times k$ subblock of $A_{i}^{-1}$.

\section{Spline Embedding}

\subsection{Global Embedding}

In Section 2, we discuss how to map the data points in each local neighborhood into a single global coordinate system. Now our task is to assemble the local treatments together to obtain an optimal global embedding $\mathcal{Y}=\left\{\boldsymbol{y}_{i}\right\}_{i=1}^{n}$.

For $k$ data points $\left\{\boldsymbol{x}_{i_{j}}\right\}_{j=1}^{k}$ in each $\mathcal{N}_{i}, i=1, \cdots, n$, we first calculate their local coordinates $\left\{\boldsymbol{t}_{i}^{j}\right\}_{j=1}^{k}$ according to the method introduced in subsection 2.2.

When mapping such $k$ local coordinates into a single global coordinate system, we get a reconstruction error $e_{i}$ as formulated in eq. (13). Finally, we can obtain the following global coordinate reconstruction error:

$$
E(Y)=\sum_{i=1}^{n} e_{i}=\sum_{i=1}^{n} \operatorname{tr}\left(Y_{i} B_{i} Y_{i}^{T}\right)
$$

Further let $Y=\left[\boldsymbol{y}_{1}, \cdots, \boldsymbol{y}_{n}\right] \in \mathbb{R}^{d \times n}$ and $S_{i} \in \mathbb{R}^{n \times k}$ be a 0 -1 selection matrix such that $Y_{i}=Y S_{i}$. Then we have

$$
E(Y)=\sum_{i=1}^{n} \operatorname{tr}\left(Y S_{i} B_{i} S_{i}^{T} Y^{T}\right)=\operatorname{tr}\left(Y S B S^{T} Y^{T}\right)
$$

where $S=\left[S_{1}, \cdots, S_{n}\right] \in \mathbb{R}^{n \times n k}$ and $B=\operatorname{diag}\left(B_{1}, \cdots, B_{n}\right) \in \mathbb{R}^{n k \times n k}$.

We use $E(Y)$ as the objective function. To avoid degenerate solutions, we add a constraint $Y Y^{T}=I$. Then, the minimum of $E(Y)$ for the $d$-dimensional global embedding can be obtained from the $d$ eigenvectors of symmetrical sparse matrix $S B S^{T}$, which are associated to $d+1$ smallest eigenvalues. We leave out the eigenvector corresponding to eigenvalue 0 and use the next $d$ eigenvectors to construct the matrix $Y$. Finally, we obtain a $d$-dimensional global embedding for $n$ scattered data points $\boldsymbol{x}_{i}, i=1,2, \cdots, n$. 


\subsection{Extension for Out-of-Sample Data Points}

Given a low-dimensional embedding $\mathcal{Y}=\left\{\boldsymbol{y}_{i}\right\}_{i=1}^{n}$ of $n$ data points $\mathcal{X}=\left\{\boldsymbol{x}_{i}\right\}_{i=1}^{n}$. Now the task is to embed a new data point $\boldsymbol{x} \in M$.

Let $\mathcal{X}^{\prime}=\mathcal{X} \cup\{\boldsymbol{x}\}$. We first construct a neighborhood $\mathcal{N}_{x}$ from $\mathcal{X}^{\prime}$. Without loss of generality, we let $\mathcal{N}_{x}=\left\{\boldsymbol{x}, \boldsymbol{x}_{1}, \cdots, \boldsymbol{x}_{k}\right\}$, with $\boldsymbol{x}_{j} \in \mathcal{X}, j=1, \cdots, k$.

For $k+1$ data points in $\mathcal{N}_{x}$, then we calculate their local coordinates and denote them by $\boldsymbol{t}, \boldsymbol{t}_{1}, \cdots, \boldsymbol{t}_{k}$. Finally, according to the $k$ known coordinates $\boldsymbol{y}_{j} \in$ $\mathcal{Y}, j=1, \cdots, k$, we re-construct a vector function $g$ such that $\boldsymbol{y}_{j}=g\left(\boldsymbol{t}_{j}\right)$. Based on $g$, we can map $\boldsymbol{t}$ into the $d$-dimensional global coordinate system and thus embed the new data point $\boldsymbol{x}$ into the learned manifold.

\section{Experimental Results}

In this section, we validate spline embedding and its extension for out-of-samples, using synthetic data points and several real-world image data sets.

Fig. 1(a) shows $n=1000$ data points sampled from a Swiss roll surface. The parameter domain of this surface is a $2 \mathrm{D}$ rectangular segment. The learned results by spline embedding using $k=12$ nearest neighbors are shown in Fig. 1(b). The color coding shows that it yields a faithful embedding. Fig. 1(c) and Fig. 1(d) demonstrate another experiment. The 1000 data points are also sampled from the same surface, but with a hole. As can be seen that the topology is well learned. Fig. 1(e) shows 1500 data points on a S-surface with four holes. Among those data points, only 750 data points are randomly selected from the half part of the whole S-surface, in which two holes are cut. The rest 750 points are generated deterministically according to the symmetry of the surface. The results learned by spline embedding with $k=12$ are shown in Fig. 1(f). We can see the learned $2 \mathrm{D}$ data points are roughly symmetrical about the line in the middle.

Fig. 2(a) shows the results of spline embedding which is applied to $n=1965$ grayscale images of faces, with $k=12$. The size of the images is $28 \times 20$. Thus the dimensionality of the data is 560 . Representative faces are given to illustrate the poses and facial expressions. The results in Fig. 2(b) are learned from $n=698$ images of a $3 \mathrm{D}$ statue, with $k=12$. Each image includes $64 \times 64$ grayscale pixels. The manifold is embedded in $\mathbb{R}^{4096}$. Representative images are shown to illustrate the lighting conditions and the camera directions. The results in Fig. 3(c) are learned from $n=400$ color teapot images [10, with $k=5$. The size of the images is $76 \times 101$. Thus the manifold is embedded in $\mathbb{R}^{23028}$. The results roughly spread along a circle, which reveal the rotations of the teapot.

Now we demonstrate an example of embedding out-of-sample data points. The original data points are taken from a group of synthetic images with $64 \times 64$ pixels. Each such image contains a $16 \times 16$ white square (see Fig. 3(a)).

First, we let the $x$ and $y$ coordinates of the center of the white square vary from 8 to 56 , both with translation step $t_{x}=t_{y}=2$. We get 576 images for training. Fig. 3(b) shows the 2D embedding results for these images, with $k=12$.

Then, we generate 576 new samples, changing $x$ and $y$ from 9 to 57 , also with step $t_{x}=t_{y}=2$. Based on the learned results as shown in Fig. 3(b), such 576 


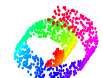

(a)

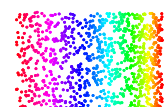

(b)

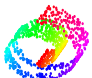

(c)

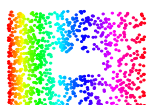

(d)

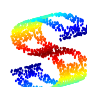

(e)

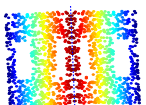

(f)

Fig. 1. (a). the 1000 data points; (b). 2D embedding results of the data points shown in (a); (c). the 1000 data points; (d). 2D embedding results of the data points in (c); (e). the 1500 data points; (f). 2D embedding results of the data points in (e)

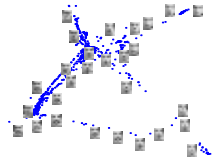

(a)

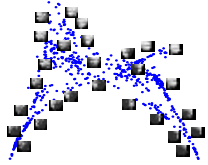

(b)

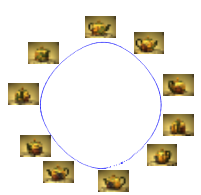

(c)

Fig. 2. (a). 2D embedding of 1965 graysacle face images; (b). 2D embedding of 698 graysacle statue images; (c). 2D embedding of 400 color teapot images

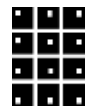

(a)

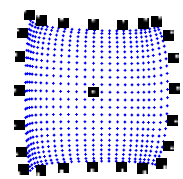

(b)

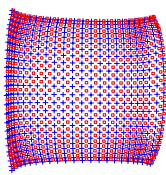

(c)

Fig. 3. (a). Examples of the synthetic binary images; (b). 2D embedding of 576 training images; (c). The embedded 576 out-of-samples, indicated by (red) square points

new data points are embedded one-by-one by using our method. The results are demonstrated in Fig. 3(c), indicated by the (red) square points. As can be seen that the new points are all faithfully embedded into the right positions.

\section{Discussion and Conclusion}

Like other manifold learning algorithms, such as Isomap, LLE, Laplacian Eigenmap, LTSA, HLLE, etc., it is necessary for us to select a proper $k$ to explore the locally geometrical structures. Generally, $k$ is not too small and too big since manifold is a combination of locally linear patches. If the data points are densely sampled from the manifold, we can take a bigger $k$.

To embed new data points, it needs smoothing splines. To obtain a $C^{p}$ continuous spline, it requires $2 s-d>p$. Given $s$ and $d$, there have $l=(d+s-$ $1) ! /(d !(s-1) !)$ terms in the polynomial. Thus the number of the nearest neighbors $k$ should be equal to or greater than $l$. In case of $s=2$ and $d=2$, it needs $k \geq 3$. To learn a global manifold embedding from a set of given data points, we can take the second term in eq. (8) as a linear polynomial. 
Compared with LTSA, our method can achieve nonlinear alignments during coordinate mapping. In computation, it needs to construct the coefficient matrix $A$ in eq. (10). The computational complexity is quadratic in the number of neighbors $k$. The complexities of other computations are roughly same as those of LTSA. A 2D embedding of $n=1500$ data points in $\mathbb{R}^{3}$ by spline embedding with $k=12$ takes about 10.1 s on a $1.7 \mathrm{GHz}$ CPU using Matlab. For the same task, LTSA needs about 9.2s, while HLLE needs about 92.5s.

In this paper, we propose a new nonlinear dimensionality reduction algorithm, spline embedding, which is developed from compatible mappings. Splines are used to construct such mappings. A global embedding is finally achieved by minimizing the low-dimensional global reconstruction error. Due to the spline interpolation, the proposed methods can be naturally extended to deal with outof-samples. In further, we would like to study semi-supervised spline embedding.

\section{Acknowledgements}

This work is supported by the Projection (60475001) of the National Nature Science Foundation of China. The anonymous reviewers have helped to improve the representation of this paper.

\section{References}

1. Tenenbaum, J. B., de Silva, V., Langford, J. C.: A global geometric framework for nonlinear dimensionality reduction. Science. 290 (2000) 2319-2323

2. Roweis, S. T., Saul, L. K.: Nonlinear dimensionality reduction by locally linear embedding. Science. 290 (2000) 2323-2326

3. Belkin, M., Niyogi, P.: Laplacian eigenmaps for dimensionality reduction and data representation. Neural Computation. 15 (2003) 1373-1396

4. Donoho, D. L., Grimes, C. E.: Hessian eigenmaps: locally linear embedding techniques for highdimensional data. Proceedings of the National Academy of Arts and Sciences. 100 (2003) 5591-5596

5. Zhang, Z.Y., Zha, H.Y.: Principal manifolds and nonlinear dimensionality reduction via tangent space alignment. SIAM Scientific Computing. 26 (2004) 313-338

6. Bengio, Y., Paiement, J., Vincent, P.: Out-of-sample extensions for LLE, Isomap, MDS, Eigenmaps and spectral clustering. Advances in Neural Information Processing Systems 16. Cambridge, MA: MIT Press (2004)

7. Duchon, J.: Splines minimizing rotation-invariant semi-norms in Sobolev spaces. In: Schempp, W., Zeller, K. (eds): Constructive Theory of Functions of Several Variables. Berlin: Springer-Verlag (1977) 85-100

8. Wahba, G.: Spline models for observsatonal data. CBMS-NSF Regional Conference Series in Applied Mathematics. SIAM Press (1990)

9. Bookstein, F. L.: Principal warps: thine-plate splines and the decomposition of deformations. IEEE Transactions on PAMI. 11(1989) 567-585

10. Weinberger, K. Q., Saul, L. K.: Unsupervised learning of image manifolds by semidefinite programming. In: CVPR. Washington DC, USA (2004) 988-995 